\title{
Converging electrophysiological evidence for a processing advantage of social over nonsocial feedback
}

\author{
Daniela M. Pfabigan ${ }^{1,2}$ (D) Shihui Han ${ }^{1}$ \\ Published online: 16 July 2019 \\ (C) The Psychonomic Society, Inc. 2019
}

\begin{abstract}
We recently demonstrated a processing advantage of social versus nonsocial feedback stimuli in a western sample by assessing phase-locked neural responses. The current study extended our previous findings to another cultural sample (Chinese) to further test whether non-phase-locked neural oscillations also exhibit the social feedback processing advantage. Fifty-three Chinese volunteers performed a time estimation task with social and nonsocial feedback stimuli (matched for complexity) while electroencephalogram was recorded. Almost entirely replicating our previous results, feedback ERPs showed a processing advantage for social compared with nonsocial stimuli. Importantly, non-phase-locked oscillations also revealed this pattern. Frontal midline theta $(\mathrm{FM} \Theta)$ oscillations differentiated between negative and positive feedback to a larger extent in response to social compared with nonsocial feedback. The current findings imply a rather universal effect of social stimulus characteristics during feedback processing and further corroborate the notion of social content as a distinct stimulus category.
\end{abstract}

Keywords Performance monitoring $\cdot$ Social content $\cdot$ Stimulus saliency $\cdot$ FRN $\cdot$ P300 $\cdot$ Frontal midline theta

\section{Introduction}

Continual monitoring of others' opinions and behaviors in relation to our own is necessary for successful social interactions, because these monitoring processes lead to behavioral adaptations when required by the situation at hand. During everyday performance monitoring, we encounter a variety of social and nonsocial (feedback) signals that provide information as to whether our behavior is appropriate. Several recent studies have demonstrated that early brain responses reflecting the activity of our performance monitoring system are sensitive to physical stimulus properties of external feedback

Electronic supplementary material The online version of this article (https://doi.org/10.3758/s13415-019-00737-9) contains supplementary material, which is available to authorized users.

Daniela M. Pfabigan

daniela.pfabigan@pku.edu.cn

1 School of Psychological and Cognitive Sciences, PKU-IDG/ McGovern Institute for Brain Research, Beijing Key Laboratory of Behavior and Mental Health, Peking University, 52 Haidian Road, Beijing 100871, China

2 Department of Behavioural Sciences in Medicine, Institute of Basic Medical Sciences, Faculty of Medicine, University of Oslo, Sognsvannsveien 9, 0372 Oslo, Norway stimuli. While Liu, Nelson, Bernat, \& Gehring (2014) and Pfabigan, Sailer, \& Lamm (2015) showed that perceptual properties such as similarity of perceptual features and stimulus size differentially influence performance monitoring correlates, we demonstrated that social versus nonsocial stimulus characteristics were reflected in early brain responses to feedback stimuli (Pfabigan, Gittenberger, \& Lamm, 2019). The authors reported that social (vs. nonsocial) and complex (vs. noncomplex) feedback stimuli enhanced processing of feedback information at several successive stages. Thus, the mere presentation of stimulus characteristics implying a social setting was enough to modulate neural correlates of feedback processing - most likely reflecting a bottom-up effect of the social stimuli. However, we demonstrated these results only in a western sample (Pfabigan, et al., 2019), which limits generalizability to this cultural group.

The influence of culture is omnipresent in our daily lives and an increasing number of findings have demonstrated that culture influences overt behavior and underlying cognitive processes and neural correlates (for an overview see Han, 2017; Han et al., 2013). The influence of social stimulus characteristics on feedback processing might be particularly strong in cultures emphasizing connectedness between the self and significant others. East Asian individuals often are characterized to view and define their self in relation to others - known as interdependent self-construal style. In contrast, western 
individuals often are characterized as being more autonomous defining their self rather independently from others-known as independent self-construal style (Markus \& Kitayama, 1991). Indeed, several studies reported a mediating role of interdependent self-construal and culture during performance monitoring in a social context (Hitokoto, Glazer, \& Kitayama, 2016; Kitayama \& Park, 2014; Pfabigan et al., 2018).

The current study was designed to replicate systemically and potentially extend our previous findings (Pfabigan, et al., 2019) in a Chinese sample (as a subgroup of East Asian individuals) characterized by an emphasis on interdependent selfconstruals. Replication studies can be seldom found in published research, although reproducibility is a basic and indispensable requirement of empirical science that should be more strongly acknowledged also in psychological science (GinerSorolla, 2012; Schmidt, 2009) to foster recent replication efforts (Open Science, 2015; Pashler \& Harris, 2012).

The second goal of the current study pertained to a methodological aspect of the question of whether social stimulus characteristics influence neural correlates of performance monitoring. Previous research mostly applied the eventrelated potential (ERP) technique in this regard, which, however, provides only phase-locked information of the ongoing EEG. In contrast, time-frequency decomposition allows the assessment of rhythmic activity, which is modulated by a mixture of phase-locked and nonphase-locked activity in response to the eliciting event (Pfurtscheller \& Lopes da Silva, 1999). Time-frequency analysis might provide more comprehensive insights into underlying neural processes than classical ERP analysis alone (Cohen, 2011; Makeig, Debener, Onton, \& Delorme, 2004). In particular in the domain of performance monitoring and cognitive control, considerable advances have been made in the past years (Cavanagh \& Frank, 2014; Cavanagh \& Shackman, 2015). Consequently, we tested whether neural oscillations corroborate or even extend previous ERP findings regarding the processing advantage of social versus nonsocial feedback stimuli.

To address our first question, we assessed three different ERP components. The first one was the Feedback-Related Negativity peaking within 200-300 ms after feedback onset (FRN; Miltner, Braun, \& Coles, 1997) at fronto-central electrode sites. Its negative deflection is more pronounced for unfavorable compared with favorable, unexpected compared with expected, and salient compared with nonsignificant feedback outcomes (Alexander \& Brown, 2011; Gehring \& Willoughby, 2002; Miltner, et al., 1997; Pfabigan, Alexopoulos, Bauer, Lamm, \& Sailer, 2011a; Pfabigan, Alexopoulos, Bauer, \& Sailer, 2011b). Recent research describes FRN amplitude variation as an unsigned prediction error signal indicting the surprise of unexpected events (Hayden, Heilbronner, Pearson, \& Platt, 2011; Talmi, Atkinson, \& El-Deredy, 2013). As the second ERP, we assessed the positive-going P300 component, which usually occurs within 300-500 ms after feedback onset with a peak at parietal electrode sites (Duncan Johnson \& Donchin, 1977; Polich, 2007), reflecting more elaborate stimulus processing of motivational saliency and context updating in working memory (Bellebaum \& Daum, 2008; Nieuwenhuis, AstonJones, \& Cohen, 2005). As the third ERP, the frontal P2 component peaking around $180 \mathrm{~ms}$ after feedback onset was assessed, which is assumed to reflect early stages of attention capture and affective significance of target stimuli (Cuthbert, Schupp, Bradley, Birbaumer, \& Lang, 2000; Potts, 2004; Potts, Martin, Burton, \& Montague, 2006).

To address the second research question, we captured frontal midline theta $(\mathrm{FM} \Theta$ ) oscillations (approximately 4-8 Hz) as nonphase-locked neural correlates of performance monitoring. FM $\Theta$ oscillations are usually captured over fronto-central electrodes and have been proposed to be sensitive to the same experimental manipulations as error-, feedback-, and conflictrelated ERP components (Cavanagh, Zambrano-Vazquez, \& Allen, 2012; Cohen, Elger, \& Ranganath, 2007). The midcingulate cortex (MCC) has been proposed as their neuronal origin (Cavanagh \& Frank, 2014). Recent research suggests that FM $\Theta$ might be a key neural mechanism of the medial prefrontal cortex (mPFC) to guide cognitive control processes (Cavanagh \& Frank, 2014; Cavanagh, et al., 2012). FM $\Theta$ oscillations are assumed to indicate the requirement of top-down control in a given situation. They might reflect the underlying neural mechanism by which top-down control is computed and communicated across broader neural networks (Cavanagh \& Frank, 2014; Cavanagh, et al., 2012). Supporting this notion, enhanced FM $\Theta$ oscillations have been associated with enhanced cognitive performance (Cohen \& Donner, 2013) and more generally with executive functioning during error and feedback processing (Enriquez-Geppert, Huster, Figge, \& Herrmann, 2014).

Participants performed the established time estimation task (Miltner, et al., 1997), which allowed approximately comparable presentation numbers of positive and negative feedback stimuli. Thumbs up/down served as social feedback stimuli, while patterned plus/minus symbols served as nonsocial ones - all representing highly overlearned stimuli conveying valence information. Importantly, these stimuli were approximately matched for visual complexity, which was identified as another factor influencing performance monitoring ERPs (Pfabigan, et al., 2019).

We hypothesized that social feedback stimuli also would demonstrate their processing advantage compared with nonsocial ones in the Chinese sample-reflected in enhanced FRN, P300, and P2 amplitudes for social compared with nonsocial feedback stimuli (Pfabigan, et al., 2019). Moreover, because previous research has shown individual variations of brain activity related to variation of interdependent selfconstrual (Ma et al., 2014), a cultural trait that characterizes East Asian samples, we explored whether interdependent self- 
construal was related to the hypothesized processing advantage of social versus nonsocial feedback stimuli (Hitokoto, et al., 2016; Kitayama \& Park, 2014). Based on previous research suggesting comparable underlying mechanisms of the investigated ERPs and neural oscillations, we assumed that FM $\Theta$ oscillations would also be enhanced for social versus nonsocial feedback stimuli (Cavanagh \& Frank, 2014).

\section{Methods}

\section{Participants}

Fifty-six volunteers participated in the electroencephalography (EEG) study. Three of them were excluded due to data acquisition problems or excessive alpha activity artefacts. The remaining 53 participants ( 29 women) were on average aged 21.5 years (standard deviation $[S D]=2.21$; range $18-26)$. Sex/ gender was equally distributed in the sample $\left(\chi_{(1)}^{2}=0.47, p=\right.$ 0.492); no age differences were observed between women and men $(t(51)=1.62, p=0.111)$. All participants were righthanded as assessed with a questionnaire ( $\mathrm{Li}, 1983)$, had normal or corrected-to-normal vision, and reported no prior or current psychiatric or neurological disorder. Written informed consent was obtained before the experiment. The study was conducted in accordance with the Declaration of Helsinki $\left(7^{\text {th }}\right.$ revision, 2013) and was approved by the Ethics Board of the School of Psychological and Cognitive Sciences, Peking University. Sample size was based on a priori power consideration relying on our previous study (Pfabigan, et al., 2019). It suggested that a sample size of at least 46 participants has sufficient power $(1-\beta>0.80)$ to detect the smallest social content effect of the previous study $\left(\eta_{p}{ }^{2}=0.20\right.$; effect size specification after Cohen (1988) using GPower 3.1; Faul, Erdfelder, Buchner, \& Lang, 2009). We recruited ten additional participants to be prepared for potential dropouts.

Before EEG data collection, participants filled in the selfconstrual scale (SCS; Singelis, 1994) to assess their dispositional independent and interdependent self-construal styles. The SCS comprises 30 items (15 per scale), which have to be rated on a 7-point Likert scale ranging from 1 (strongly disagree) to 7 (strongly agree). The average ratings for independent and interdependent self-construals were calculated per participant to acknowledge orthogonality of the two constructs. Larger values represent more pronounced independence and interdependence.

\section{Time estimation task}

In a modified version of the time estimation task (Miltner, et al., 1997; Pfabigan, Sailer, \& Lamm, 2015; Pfabigan, Zeiler, Lamm, \& Sailer, 2014), participants were required to estimate the passing of one second via button press. The trial structure was as follows: first, a central black fixation dot on a grey screen was shown for 1,000 ms. Afterwards, a black star replaced the dot for $250 \mathrm{~ms}$, which started the time estimation of the respective trial. During the subsequent 1,750 ms (blank grey screen), participants could indicate the estimated elapse of 1 second via pressing button 1 on a standard keyboard with their right index finger; 2,000 ms after star onset, performance feedback was presented for 1,000 ms. The following intertrialinterval (black fixation dot) varied randomly between 1,4001,600 ms. Feedback was always provided based on individual performance. However, task difficulty was adjusted to the individual performance level with a staircase procedure to guarantee approximately comparable numbers of correct and incorrect trials. At the beginning, positive feedback was given in cases a button press fell in the time window of 900 to $1,100 \mathrm{~ms}$ after star onset. Subsequently, the size of this time window was adjusted based on individual performance in the preceding trial (Miltner, et al., 1997). After a trial with positive feedback (i.e., a correct time estimation), the time window was narrowed down by $10 \mathrm{~ms}$ at both ends of the window. After a trial with negative feedback (i.e., an incorrect time estimation), the time window became wider again by adding $10 \mathrm{~ms}$ at both ends. Consequently, the overall probability of positive and negative feedback was approximately $50 \%$ for all participants.

Social feedback stimuli consisted of naturalistic hand gestures of "thumbs up" or "thumbs down" (indicating either positive or negative feedback). Nonsocial feedback stimuli consisted of black patterned "+" or "-" symbols (indicating either positive or negative feedback). Based on our previous results, we used only stimuli for the current study that were considered complex stimuli (Pfabigan, et al., 2019). Thumbs up/down stimuli were presented in an allocentric perspective to emphasize that feedback is given externally and serves an evaluative function. Stimuli were presented on a grey background and were comparable in size and luminance (please refer to Pfabigan, et al., 2019, for further details on objective and subjective stimulus characteristics, and to Fig. 1 for an illustration of the stimuli). Participants were explicitly instructed that they should consider social and nonsocial feedback stimuli as equivalent indicators of their time estimation accuracy.

The experiment consisted of ten training trials and 200 experimental trials. The respective 100 trials depicting either social or nonsocial stimuli were presented block-wise as previously suggested (Pfabigan, et al., 2014). Block order was counterbalanced across participants. Overall EEG data collection lasted approximately 20 minutes. Afterwards, participants rated the presented feedback stimuli regarding motivational significance (not motivating vs. very motivating) and arousal (very calm vs. very exciting) on 7-point Likert scales. They performed another experimental task afterwards, which is not in the scope of the current report. At the end of the experiment, participants received a financial remuneration of RMB 100 for their participation. 


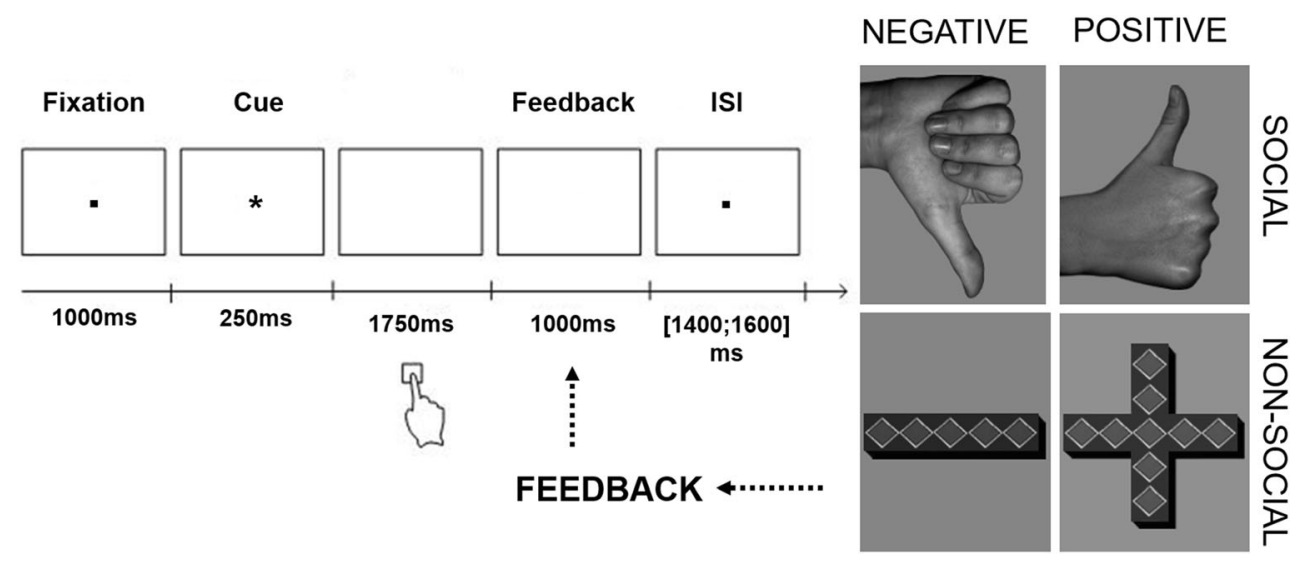

Fig. 1 Trial timing is depicted on the left side of the figure, while the four different feedback stimuli are illustrated on the right side

\section{EEG acquisition and analyses}

Stimulus presentation was controlled by E-Prime 2.0 (Psychology Software Tools, Inc., Sharpsburg, PA). Participants were seated in a sound-attenuated, shielded room approximately $90 \mathrm{~cm}$ in front of a 19" TFT monitor (AOC G2460p LED). EEG was recorded via 64 electrodes embedded in a fabric electrode cap (EASYCAP GmbH, Herrsching, Germany; 10-20 system, 64Ch Standard Brain cap, Asian fit). One electrode was placed $1 \mathrm{~cm}$ below the left eye to assess vertical eye-movements. EEG signals were collected with a time constant of 10 seconds up until $1,000 \mathrm{~Hz}$, referenced online against $\mathrm{FCz}$, whereas $\mathrm{AFz}$ served as ground electrode (BrainAmp DC; Brain Products GmbH, Gilching, Germany).

\section{Behavioral data analysis}

Differences in response times were calculated per participant and per condition between each trial and its preceding trial to describe changes in response times evoked by directly preceding feedback. These mean trial-to-trial changes were further separated for trials in which reaction time changes were classified as correct adjustments (i.e., the difference in estimation time between the current trial (n) and the previous trial (n-1) was larger than the difference between estimation time in trial (n) and the 1,000-ms goal) and those classified as incorrect adjustments (i.e., the difference in estimation time between the current trial (n) and the previous trial (n-1) was smaller than the difference between estimation time in trial (n) and the 1,000-ms goal; Pfabigan, et al., 2015). These changes in response time were subjected to a $2 \times 2 \times 2$ repeated-measures ANOVA with the within-subject factors social content (social, nonsocial), feedback valence (negative, positive), and estimation adjustment (incorrect, correct) to investigate time estimation changes as a function of feedback conditions. Please refer to Supplementary Materials, chapter 1.7, for an alternative assessment of changes in time estimation behavior. In the current study, we introduced an additional measure of time estimation performance. We extracted the average time interval length during which an estimation was considered as correct to assess estimation quality. The interval length was subjected to a paired-samples $t$-test comparing social versus nonsocial feedback trials. Post-experimental ratings concerning motivational significance and arousal were analysed separately using $2 \times 2$ repeated measures ANOVAs with the withinsubject factors social content and feedback valence.

\section{EEG preprocessing}

EEG data were analysed using EEGLAB 13_1_1b (Delorme \& Makeig, 2004) implemented in Matlab R2014a. Data analyses were performed as parallel to our previous study as possible (Pfabigan, et al., 2019). A high pass $(0.1 \mathrm{~Hz})$ and a lowpass filter (cut-off frequency $30 \mathrm{~Hz}$, roll-off $6 \mathrm{~dB} /$ octave) were applied offline to the EEG data. Channel $\mathrm{FCz}$ was reinstated, subsequently data were re-referenced to linked mastoids and extended infomax independent component analysis (ICA; Bell \& Sejnowski, 1995) was applied to detect eye movement-related artefacts. After discarding these artefacts (on average 2.5 ICs per participant, $\mathrm{SD}=0.93$, range: $1-5$ ), data segments of the four possible conditions were extracted starting 2,200 ms before feedback onset and lasting for $3,300 \mathrm{~ms}$ (social positive [Spos], social negative [Sneg], nonsocial positive [NSpos], and nonsocial negative [NSneg]). Subsequently, semiautomatic artefact correction was performed in EEGLAB. At first, trials with voltage values exceeding $\pm 75 \mu \mathrm{V}$ (function pop_eegthres) or voltage drifts $>50 \mu \mathrm{V}$ (function pop_eegrejtrend) were automatically marked by the algorithms. These trials were rejected in case visual inspection also indicated the presence of artefacts. Artefact-free segments were averaged participant- and condition-wise; on average $35.85(\mathrm{SD}=8.52)$ trials per condition. 


\section{ERP analysis}

The $\mathrm{P} 2$ component was scored as the most positive peak in the time window 130-230 ms after feedback onset. The FRN component was scored as peak-to-peak difference measure (P2 minus FRN component; Holroyd, Nieuwenhuis, Yeung, \& Cohen, 2003; Pfabigan, Alexopoulos, Bauer, \& Sailer, 2011b) to account for component overlap with the preceding P2 component. FRN peak amplitudes (interval 200-300 ms after feedback onset) and the amplitude values of the preceding positive P2 peak were extracted for all conditions at a fronto-central electrode cluster centred around $\mathrm{FCz}$ (the cluster comprised of electrodes $\mathrm{F} 1, \mathrm{Fz}, \mathrm{F} 2, \mathrm{FC} 1, \mathrm{FCz}, \mathrm{FC} 2, \mathrm{C} 1, \mathrm{Cz}, \mathrm{C} 2$ ) to increase signal-to-noise ratio and to keep the statistical models as simple as possible (as suggested by Luck and Gaspelin, 2017). The P300 component also was assessed as peak-to-peak measure to account for component overlap with the preceding negative ERP (N2 minus P300 component; Pfabigan, Alexopoulos, Bauer, \& Sailer, 2011b). P300 peaks (interval 250-500 ms after feedback onset) were extracted for all conditions at a parietal electrode cluster (comprising of electrodes $\mathrm{CP} 1, \mathrm{CPz}, \mathrm{CP} 2, \mathrm{P} 1$, $\mathrm{Pz}, \mathrm{P} 2)$. Furthermore, assessing peak values for the three ERP components allowed investigating their latency variation, which provided valuable information regarding stimulus processing speed. For example, P300 latencies are assumed to reflect stimulus classification demands (Kutas, McCarthy, \& Donchin, 1977). FRN, P300, and P2 peak and latency measures were analysed separately with two-way repeated-measures ANOVAs with the within-subject factors social content (social, nonsocial), and feedback valence (positive, negative). FRN and P2 peak latencies were measured from feedback onset to the corresponding FRN/P2 peak at the FCz electrode cluster; P300 peak latency from feedback onset to the corresponding P300 peak at the Pz electrode cluster.

\section{Time-frequency analysis}

We calculated trial- and electrode-wise event-related spectral perturbations (ERSPs) per participant with artefact-free trials using a complex Morlet wavelet decomposition implemented in the EEGLAB newtimefo function. Power was calculated from 4 to $20 \mathrm{~Hz}$ in 25 bins (zero-padded 3-cycle Morlet wavelets were used for the lowest frequency, increasing linearly by 0.5 cycles until the highest frequency). We used the whole time window of $-2,200$ to $1,100 \mathrm{~ms}$ around feedback onset for calculation to prevent edge effects influencing the FM $\Theta$ period. Furthermore, mean power with the time interval of -400 to $-200 \mathrm{~ms}$ before feedback onset served as baseline interval. This interval was chosen to avoid interference of windowing post-feedback activity and padding values together, which could yield biased ERSP estimates. Subsequently, power values (in $\mu \mathrm{V}^{2}$ ) were averaged over all conditions and participants. In line with previous studies, pronounced FM $\Theta$ activity was observed at fronto-central electrode sites (Cavanagh \& Frank, 2014; Cavanagh, et al., 2012; van der Veen, van der Molen, van der Molen, \& Franken, 2016) (Figure 3A). Keeping our analysis strategies as comparable as possible, we used the same electrode cluster as for FRN/P2 analysis. FM $\Theta$ responses $(4-7.2 \mathrm{~Hz})$ were extracted from baseline-corrected ERSPs in the interval 200-400 ms after feedback onset at the frontal electrode cluster subject- and condition-wise (Osinsky, Seeger, Mussel, \& Hewig, 2016). These values were analysed with a two-way repeated-measures ANOVA with the within-subject factors social content and feedback valence. To further extend the current replication, we computed an equivalent time frequency analysis with the data of the previous study. Please refer to Supplementary Material (chapter 1.6) for details and results. To explore whether dispositional selfconstrual was associated with neural correlates of performance monitoring and rating data, we calculated social content difference measures (Sneg minus NSneg; Spos minus NSpos) and tested their association with self-construal scores with Spearman correlations (Bonferroni-adjusted $p<0.0125$; Rousselet \& Pernet, 2012). We further tested whether social content difference measures of FRN amplitudes and FM $\Theta$ oscillations were related to each other using Spearmen correlations (Bonferroni-adjusted $p<0.025$ ).

Statistical analyses were performed using SPSS 22 (SPSS Inc., IBM Corporation, NY) and Statistica 6.0 (StatSoft Inc., Tulsa, OK). Significant interaction effects were explored with planned contrasts (negative minus positive) when appropriate or Tukey post-hoc tests. The significance level was set at $p<$ 0.05. Partial eta-squared $\left(\eta_{p}{ }^{2}\right)$ is reported to indicate effect sizes for significant ANOVA results (Kirk, 1996), while Cohen's $d$ is reported for $t$-tests (Cohen, 1988). A winsorisation procedure (Wilcox, 2012) was applied per condition for ERP, FM $\Theta$, and behavioral data to account for outliers before statistical analyses (see Kogler, Sailer, Derntl, \& Pfabigan, 2017; Rauchbauer, Majdandžić, Hummer, Windischberger, \& Lamm, 2015, for a detailed description). As noted by Keselman, Wilcox, \& Lix (2003) and Wilcox (2010), including outlier values in analyses violates assumptions of general linear model estimations and should thus be avoided.

To demonstrate generalizability of the current results, we assessed whether ERP and FM $\Theta$ variation and behavioral correlates were influenced by participants' sex/gender (via conducting additional analyses adding sex/gender as between-subject effect to the respective ANOVA models).

\section{Results}

\section{Behavioral results}

Table 1 summarizes the means and standard deviations of the behavioral data. The overall frequency of positive feedback during the experiment was $48.95 \%(S D=2.78)$, which reflects 
Table 1 Behavioral and rating data

\begin{tabular}{|c|c|c|c|c|}
\hline & \multicolumn{2}{|c|}{ Frequency positive feedback } & \multicolumn{2}{|c|}{ Interval length } \\
\hline & $M$ & $S D$ & $M$ & $S D$ \\
\hline non-social & 48.72 & 5.51 & 140.33 & 59.69 \\
\hline \multirow[t]{2}{*}{ social } & 49.19 & 4.66 & 129.69 & 44.59 \\
\hline & \multicolumn{2}{|c|}{ Correct adjustments } & \multicolumn{2}{|c|}{ Incorrect adjustments } \\
\hline Trial-to-trial changes (ms) & $M$ & $S D$ & $M$ & $S D$ \\
\hline non-social neg & 338.56 & 133.21 & 107.63 & 37.37 \\
\hline non-social pos & 176.81 & 73.22 & 126.41 & 48.49 \\
\hline social neg & 323.86 & 118.06 & 99.39 & 36.48 \\
\hline \multirow[t]{2}{*}{ social pos } & 171.86 & 67.94 & 113.86 & 46.22 \\
\hline & \multicolumn{2}{|c|}{ Motivational significance } & \multicolumn{2}{|l|}{ Arousal } \\
\hline Post-experimental ratings & $M$ & $S D$ & $M$ & $S D$ \\
\hline non-social neg & 3.04 & 1.71 & 3.70 & 1.49 \\
\hline non-social pos & 4.92 & 1.63 & 5.02 & 1.49 \\
\hline social neg & 3.51 & 2.17 & 4.25 & 1.87 \\
\hline social pos & 5.55 & 1.59 & 5.38 & 1.29 \\
\hline
\end{tabular}

the successful implementation of the staircase procedure. Positive feedback occurred equally often in social and nonsocial feedback conditions $(t(52)=0.40, p=0.690)$.

Trial-to-trial changes in response times were influenced by feedback valence $\left(F(1,52)=245.25, p<0.001, \eta_{p}{ }^{2}=0.83\right)$, estimation adjustment $\left(F(1,52)=287.75, p<0.001, \eta_{p}{ }^{2}=\right.$ $0.85)$, and by trend by social content $(F(1,52)=3.00, p=$ $0.089, \eta_{p}{ }^{2}=0.06$; larger changes following nonsocial than social feedback). There was a significant interaction between feedback valence and estimation adjustment $(F(1,52)=$ $\left.251.14, p<0.001, \eta_{p}{ }^{2}=0.83\right)$, the remaining interactions were not significant (all $p$ values $>0.150$ ). Post-hoc analyses revealed that trial-to-trial changes in reaction times were largest after negative feedback, yielding a correct estimation adjustment (all $p$ values $<0.001$ ). No differences in trial-to-trial changes in response times were observed between negative and positive feedback, leading to an incorrect estimation adjustment $(p=0.152)$. Correct estimation adjustments after positive feedback yielded larger trial-to-trial changes compared to both incorrect adjustments (both $p$ values $<0.001$ ), but smaller changes compared to correct adjustments after negative feedback $(p<0.001)$. The average interval length to receive correct feedback was shorter for social compared with nonsocial feedback trials $(t(52)=2.03, p=0.048, d=$ $0.19)$ indicating that participants showed higher estimation quality for social compared with nonsocial feedback. Please refer to Supplementary Materials for an alternative quantification approach for trial-to-trial changes in estimation behavior (chapter 1.7).

Post-experimental ratings of motivational significance showed significant main effects of social content $(F(1,52)=$ $\left.10.11, p=0.002, \eta_{p}{ }^{2}=0.16\right)$ and feedback valence $(F(1,52)=$ 43.06, $\left.p<0.001, \eta_{p}{ }^{2}=0.45\right)$. Their interaction was not significant $(p=0.658)$. Social and positive feedback stimuli were rated as more motivating than nonsocial and negative ones. Post-experimental arousal ratings showed significant main effects of social content $\left(F(1,52)=7.89, p=0.007, \eta_{p}{ }^{2}\right.$ $=0.13)$ and feedback valence $\left(F(1,52)=29.87, p<0.001, \eta_{p}{ }^{2}\right.$ $=0.37)$. Their interaction was not significant $(p=0.562)$. Social and positive feedback stimuli were rated as more arousing than nonsocial and negative ones.

Ratings of self-construals were as follows: SCS Independence $(M=4.62, S D=0.59)$, SCS Interdependence $(M=4.86, S D=0.64)$. Interdependent self-construal was rated significantly higher than independent self-construal $(t(52)=$ $-2.66, p=0.010, d=0.40)$. Trial-to-trial changes in response times, time estimation interval length, post-experimental ratings, and SCS scores were not influenced by participants' sex/ gender (all $p$ values $>0.075$ ). Only the frequency of positive feedback showed a social content $x$ sex/gender interaction $\left(F(1,52)=5.09, p=0.028, \eta_{p}{ }^{2}=0.09\right)$. Tukey post-hoc tests showed that men received more positive feedback during nonsocial trials than women during social trials $(p=0.031)$.

\section{EEG results}

To keep the results section concise, Table 2 shows the exact statistical values and the information as to whether the results of our previous study (Pfabigan, et al., 2019) were replicated. Please refer to Supplementary Material for corroborating results of alternative ERP quantification methods (chapters 1.1-1.5). Table 3 shows means and SD of the EEG results.

The FRN ANOVA model yielded two significant main effects and a significant interaction on FRN peak-topeak amplitudes. The interaction was driven by a larger difference between negative and positive feedback $(\Delta F R N)$ in 
Table 2 Results of the ERP ANOVA models

\begin{tabular}{|c|c|c|c|c|c|c|}
\hline & & & $F$ & $p$ & $\eta_{p}{ }^{2}$ & Replication of Pfabigan et al., 2019 \\
\hline \multirow[t]{6}{*}{ FRN } & $p 2 p$ & social content & 21.58 & $<.001$ & 0.29 & yes \\
\hline & & feedback valence & 43.00 & $<.001$ & 0.45 & yes \\
\hline & & interaction & 26.06 & $<.001$ & 0.33 & yes \\
\hline & latency & social content & 20.00 & $<.001$ & 0.28 & yes \\
\hline & & feedback valence & 43.42 & $<.001$ & 0.46 & yes \\
\hline & & interaction & 2.63 & 0.111 & 0.05 & yes \\
\hline \multirow[t]{6}{*}{ P300 } & $p 2 p$ & social content & 29.45 & $<.001$ & 0.36 & yes \\
\hline & & feedback valence & 1.43 & 0.237 & 0.03 & yes \\
\hline & & interaction & 0.51 & 0.479 & 0.01 & yes \\
\hline & latency & social content & 5.62 & 0.022 & 0.10 & no \\
\hline & & feedback valence & 99.98 & $<.001$ & 0.66 & yes \\
\hline & & interaction & 1.97 & 0.166 & 0.04 & yes \\
\hline \multirow[t]{6}{*}{ P2 } & $b 2 p$ & social content & 14.63 & $<.001$ & 0.22 & yes \\
\hline & & feedback valence & 50.78 & $<.001$ & 0.49 & yes \\
\hline & & interaction & 40.67 & $<.001$ & 0.44 & yes \\
\hline & latency & social content & 18.63 & $<.001$ & 0.26 & yes \\
\hline & & feedback valence & 1.37 & 0.247 & 0.03 & yes \\
\hline & & interaction & 0.82 & 0.370 & 0.02 & yes \\
\hline
\end{tabular}

$\mathrm{df}=1,52$ for all models; $\mathrm{p} 2 \mathrm{p}=$ peak-to-peak assessment of peaks; $\mathrm{b} 2 \mathrm{p}=$ baseline-to-peak assessment of peaks; bold font indicates significant results

social $(M=4.37 \mu \mathrm{V}, S D=3.55)$ than nonsocial stimuli $(M=$ $0.58 \mu \mathrm{V}, S D=4.18 ; t(52)=-5.10, p<0.001, d=0.86)$. The ANOVA of FRN latencies yielded two significant main effects, i.e., the FRN component peaked earlier for social than nonsocial, and for positive than negative feedback.

The P300 ANOVA model yielded a significant main effect of social content. P300 amplitudes were more positive following social compared with nonsocial feedback. The P300 latency ANOVA model yielded two significant main effects. P300 peaks were observed earlier for social than nonsocial and for positive than negative feedback.

The P2 ANOVA model yielded two significant main effects and a significant interaction. Tukey post-hoc tests showed that $\mathrm{P} 2$ amplitudes were smallest after nonsocial negative feedback (both $p$ values $<0.001)$; the other conditions did not differ from each other (all $p$ values $>0.998$ ). The P2 latency ANOVA model yielded only a significant main effect of social content. P2 amplitudes peaked earlier after social than nonsocial feedback.

Neither P2, FRN, nor P300 amplitudes were influenced by participants' sex/gender (all $p$ values $>0.110$ ). See Fig. 2 for EEG results.

The FM $\Theta$ oscillations ANOVA model (mean FM $\Theta$ power 200-400 ms after feedback onset) yielded significant main effects for social content $(F(1,52)=4.54, p=0.038$, $\left.\eta_{p}{ }^{2}=0.08\right)$ and feedback valence $(F(1,52)=36.88, p<$ $\left.0.001, \eta_{p}{ }^{2}=0.42\right)$ and their interaction $(F(1,52)=13.02, p=$ $\left.0.001, \eta_{p}{ }^{2}=0.20\right)$. Tukey post-hoc tests showed that social negative feedback elicited enhanced FM $\Theta$ than nonsocial negative feedback $(p<0.001)$, whereas social and nonsocial positive feedback did not differ regarding $\operatorname{FM} \Theta(p=0.995)$. Moreover, the difference between negative and positive feedback stimuli $(\triangle \mathrm{FM} \Theta)$ was larger for social $\left(\mathrm{M}=1.13 \mu \mathrm{V}^{2}\right.$, $\mathrm{SD}=1.10)$ than for nonsocial feedback $\left(\mathrm{M}=0.46 \mu \mathrm{V}^{2}, \mathrm{SD}=\right.$ $1.22 ; t(52)=-3.61, p<0.001, d=0.58)$. See Fig. 3 for FM $\Theta$ results.

Adding sex/gender to the ANOVA model, a social content $x$ sex/gender interaction was observed $(F(1,52)=5.76, p=$ $\left.0.020, \eta_{p}{ }^{2}=0.10\right)$ in addition to the social content $x$ valence interaction. Tukey post-hoc tests showed that FM $\Theta$ power was higher for social compared with nonsocial stimuli in women $(p=0.010)$, whereas men showed no differences ( $p$ $=0.992)$. The three-way interaction was not significant ( $p=$ $0.424)$.

\section{Correlation analyses}

We observed no significant correlations between independent or interdependent self-construal scores and difference scores of ERP data (all $p$ values $>0.110$ ), FM $\Theta$ power (all $p$ values $>0.104$ ), or rating data (all $p$ values $>0.173$ ). FRN amplitudes and $\mathrm{FM} \Theta$ power were not significantly correlated with each other for negative $\left(r_{s}=0.229, p=0.099\right)$ and positive $\left(r_{s}=0.177, p=0.204\right)$ social content difference measures, suggesting a differentiation between phaselocked and nonphase-locked stimulus processing during feedback presentation. 


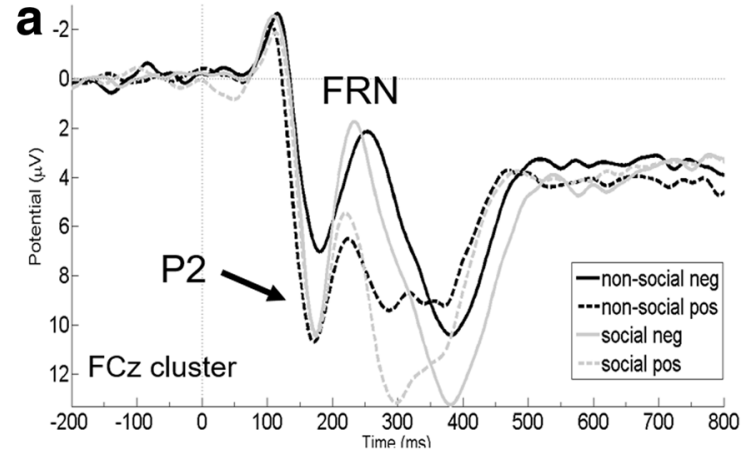

Valence difference FRN amplitudes

C

6

(negative minus positive)

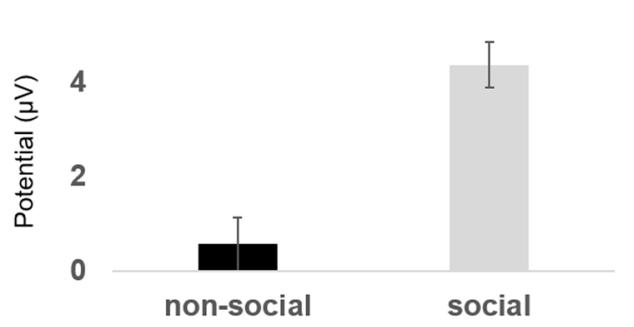

Fig. 2 (a) Amplitude courses of P2 and FRN components at the frontal cluster. (b) Amplitude course of the P300 component at the parietal cluster. (c) Bar graphs of the valence difference (negative minus positive) of FRN amplitudes for nonsocial and social feedback. (d)

\section{Discussion}

The current study was designed to replicate and extend previous findings by testing a processing advantage of social compared with nonsocial feedback in a Chinese sample. Our ERP results first replicated our previous findings almost entirely (Pfabigan, et al., 2019). We observed more pronounced amplitudes for social compared with nonsocial feedback stimuli in three successive feedback processing stages - indexed by P2, FRN, and P300 components (refer to Supplementary Materials, chapter 1.5, for a discussion on P300 amplitude variation in response to feedback valence). Moreover, the current behavioral results were in line with the previous findings. Correct adjustments of time estimation performance were preceded by negative feedback and resulted in largest changes in time estimation response times. By trend, adjustments were smaller following social stimuli. Adding a measure of estimation quality, we observed better estimations for social than for nonsocial stimuli-suggesting that the social content manipulation was indeed behaviorally relevant. As the second main goal, the current work conducted time-frequency analyses to corroborate the influence of social stimulus characteristics on neural correlates of performance monitoring. Similar to the FRN results, the difference of negative minus positive frontal midline theta oscillations (FM $\Theta$ ) was larger for social compared with nonsocial feedback stimuli. This result also was

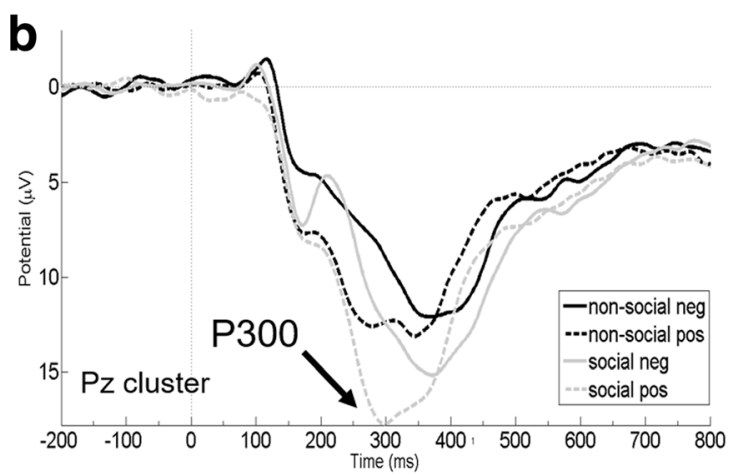

\section{Valence difference plots}

d

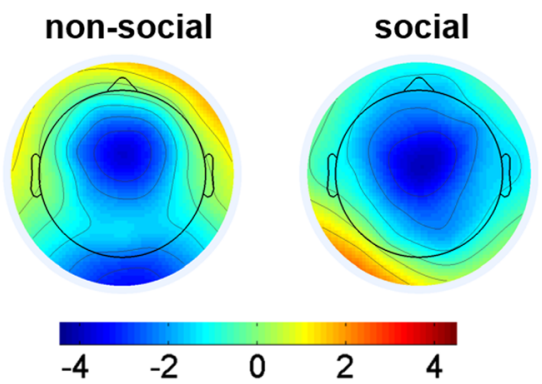

Topographical maps (in $\mu \mathrm{V}$ ) of these valence difference waves for the FRN component (FRN mean latency topography minus P2 mean latency topography per condition). Negative is drawn upward per convention. Feedback onset is at time point zero

observed when conducting a similar time-frequency analysis in the previous dataset. No significant associations of dispositional self-construal were found with neural or behavioural correlates.

Overall, the current ERP and FM $\Theta$ results corroborate the suggestion that social stimuli were processed preferentially and more elaborately compared with nonsocial ones. Time estimation quality even benefitted from using social feedback stimuli. This emphasizes again the intrinsic relevance of social stimuli during social interaction (Dunbar, 1998) and their link to guiding behavior (Hariri, Tessitore, Mattay, Fera, \& Weinberger, 2002). This pattern was similarly observed in individuals with Chinese and western cultural backgrounds for such universal social communicative gestures, such as thumbs up/down. Such culturally universal brain activation further underlines the importance of social signals. Our results corroborate the notion of previous imaging studies suggesting that social versus nonsocial information content could be seen as a distinct stimulus category (Britton et al., 2006; Norris, Chen, Zhu, Small, \& Cacioppo, 2004; Scharpf, Wendt, Lotze, \& Hamm, 2010; Vrtička, Sander, \& Vuilleumier, 2011; Wagner, Kelley, Haxby, \& Heatherton, 2016). These studies reported that social versus nonsocial information led to preferential processing in cortical and subcortical areas (e.g., occipital, frontal areas, amygdala, and insula). Thereby, it has been suggested that social versus nonsocial information 
a FMO scalp distribution - all conditions
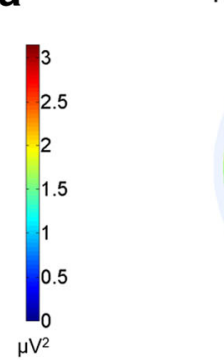

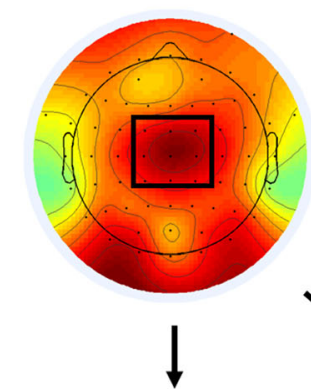

C $\mathrm{FM} \Theta$ time course at $\mathrm{FC} z$ cluster

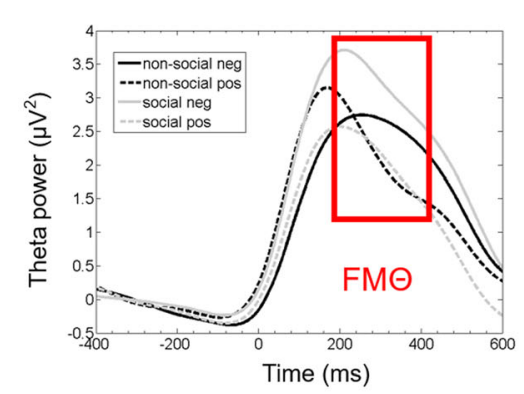

b TF decomposition - all conditions at FCz cluster

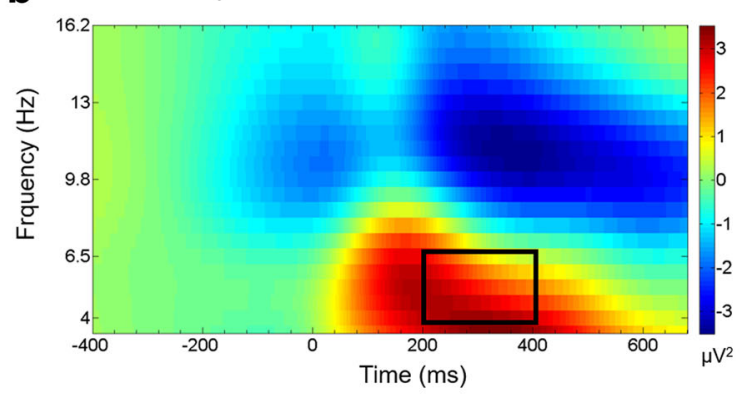

Valence difference of $\mathrm{FM} \Theta$ (neg - pos)

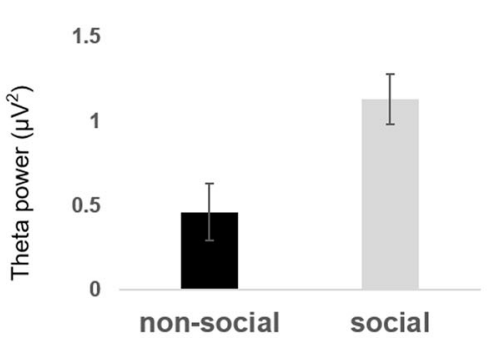

\section{e TF decomposition of valence difference (neg - pos)}

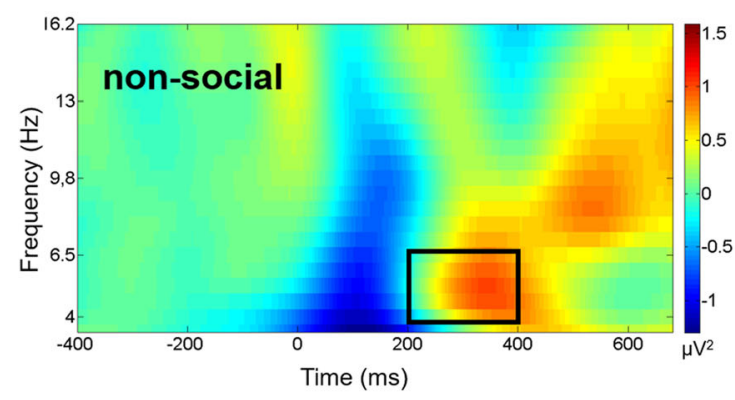

Fig. 3 (a) FM $\Theta$ scalp topography $200-400 \mathrm{~ms}$ after feedback onset averaged over all participants and conditions. The black rectangle indicates the electrode cluster around $\mathrm{FCz}$ used for further analysis. (b) Timefrequency (TF) decomposition from 4 to $16 \mathrm{~Hz}$ in a time window starting $400 \mathrm{~ms}$ before feedback onset and lasting for $1,100 \mathrm{~ms}$, averaged over all participants and conditions. The black rectangle denotes frequency and

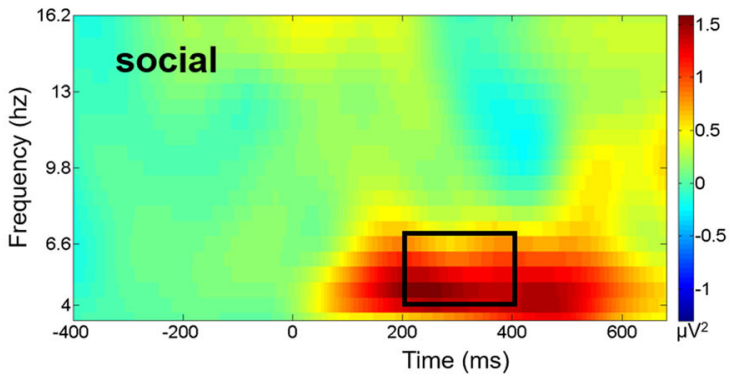

time window for FM $\Theta$ analysis. (c) FM $\Theta$ time courses in the same time window separated for the four feedback conditions. (d) Bar graphs of the valence difference (negative minus positive) FM $\Theta$ for nonsocial and social feedback. (e) Time-frequency (TF) decomposition of the valence difference for nonsocial and social feedback. The black rectangles denote frequency and time windows for FM $\Theta$

Table 3 Means and SD of ERPs and neural oscillations

\begin{tabular}{|c|c|c|c|c|c|c|c|c|}
\hline & \multicolumn{2}{|c|}{ Nonsocial neg } & \multicolumn{2}{|c|}{ Nonsocial pos } & \multicolumn{2}{|c|}{ Social neg } & \multirow{2}{*}{$\begin{array}{l}\text { Social pos } \\
M\end{array}$} & \multirow[b]{2}{*}{$S D$} \\
\hline & $M$ & $S D$ & $M$ & $S D$ & $M$ & $S D$ & & \\
\hline FRN amplitudes $(\mu \mathrm{V})$ & 8.11 & 4.47 & 7.53 & 5.01 & 12.11 & 5.47 & 7.74 & 4.55 \\
\hline FRN latency (ms) & 265.13 & 32.49 & 242.68 & 34.36 & 244.91 & 29.17 & 231.70 & 23.73 \\
\hline P300 amplitudes $(\mu \mathrm{V})$ & 8.85 & 4.39 & 8.42 & 4.37 & 12.40 & 5.73 & 11.31 & 5.30 \\
\hline P300 latency (ms) & 387.32 & 49.68 & 323.58 & 50.94 & 372.08 & 48.00 & 319.28 & 36.96 \\
\hline P2 amplitudes $(\mu \mathrm{V})$ & 8.12 & 4.36 & 12.26 & 5.86 & 12.12 & 6.34 & 12.19 & 6.14 \\
\hline P2 latency (ms) & 192.75 & 23.54 & 188.64 & 27.00 & 180.19 & 15.51 & 179.81 & 13.75 \\
\hline $\mathrm{FM} \Theta$ power $\left(\mu \mathrm{V}^{2}\right)$ & 2.61 & 1.50 & 2.15 & 1.57 & 3.25 & 1.47 & 2.12 & 1.41 \\
\hline
\end{tabular}


is processed in an additive or even interactive manner with emotional content dimensions, for which processing advantages are already established (Britton, et al., 2006; Hariri, et al., 2002; Norris, et al., 2004; Scharpf, et al., 2010; Vrtička, et al., 2011).

Furthermore, apart from our own study, recent electrophysiological evidence supports the claim of a processing advantage of social versus nonsocial information. Okruszek et al. (2016) reported a clear differentiation between social and nonsocial stimulus content for negative versus neutral complex visual scenes within the first $300 \mathrm{~ms}$ of stimulus processing. Using written stimulus material, Bayer, Ruthmann, \& Schacht (2017) also demonstrated processing differences between social and nonsocial stimulus content for negatively valenced versus neutral sentences within the first $300 \mathrm{~ms}$. Because both studies demonstrate that social content is processed at rather early stages in the processing stream, one could consider it as an important stimulus aspect. This is not surprising, because social stimuli convey powerful positive and negative social motives, such as affiliation and protection, but also disapproval and social exclusion (Baumeister, Vohs, DeWall, \& Zhang, 2007; Norris, et al., 2004; Porges, 2003). A recent study (Schacht \& Vrticka; 2018) reporting a social stimulus processing advantage also for positive stimuli suggests the component process model of emotion (Scherer, 2009) as theoretical model to explain these early social stimuli processing advantages. This theory proposes a series of relevance checks, such as novelty, intrinsic pleasantness, and goal relevance (Scherer, 2009) during stimulus evaluation. Based on their findings, Schacht \& Vrtička (2018) suggest that social stimulus content is appraised during one of the first relevance checks, possibly as aspect of intrinsic pleasantness. However, in contrast to their findings, the current study observed processing advantages of social stimuli specifically for negative stimuli (FRN and FM $\Theta$ ). This might be caused by the inherent nature of feedback stimuli, because negative outcomes are behavirally more relevant than positive ones.

As of yet, only a few studies investigated whether neural oscillations are sensitive to performance monitoring in a social context. Measuring intracranial EEG, Cristofori et al. (2013) tested whether FM $\Theta$ oscillations were responsive to a standard social exclusion situation. Indeed, the authors observed enhanced FM $\Theta$ activity in exclusion compared with inclusion trials at electrodes implanted in cortical and subcortical areas of the so-called pain matrix. This led them to conclude that FM $\Theta$ oscillations also might represent an indicator of social pain. However, exclusion trials might be more conflicting with one's own expectations than inclusion trials and therefore require enhanced cognitive control demands, which is another explanation for enhanced $\mathrm{FM} \Theta$ band activity in these trials (Cavanagh \& Frank, 2014). Controlling for the cognitive conflict confound, van Noordt, White, Wu, Mayes, \& Crowley
(2015) again observed enhanced FM $\Theta$ activity for rejection events compared with visually similar "not my turn" events in a child sample. Together, both results suggest that social exclusion trials were the most salient ones. Investigating neural oscillations with a social evaluation paradigm, van der Veen et al. (2016) and van der Molen, Dekkers, Westenberg, van der Veen, \& van der Molen (2016) observed enhanced FM $\Theta$ for unexpected social feedback. While these studies collectively demonstrate that FM $\Theta$ oscillations can serve as reliable markers of performance monitoring in a social context, no study directly investigated the influence of social versus nonsocial context or confounding variables so far. Thus, the current results extend the previous studies by demonstrating that variation in FM $\Theta$ oscillations - as a measure of phase-locked and nonphase-locked neural activation-also reflected the distinction in social and nonsocial feedback stimuli (in the absence of expectancy or cognitive conflict manipulations). This further suggests that cognitive control processes implemented by the mPFC might become more strongly engaged when social versus nonsocial stimuli are involved (Cavanagh \& Frank, 2014; Cavanagh, et al., 2012).

No significant association between FRN amplitudes and $\mathrm{FM} \Theta$ oscillations were observed in the current study. This is not unexpected, because the current time-frequency decomposition comprises a mixture of phase-locked and nonphase-locked activity while FRN amplitude variation comprises only phase-locked activity (Pfurtscheller \& Lopes da Silva, 1999).

Furthermore, we observed no reliable association of EEG measures and individual self-construal scores in the current study. This is in contrast to previous studies reporting an association between error- and feedback-related amplitude variation and interdependent self-construal scores (Hitokoto, et al., 2016; Kitayama \& Park, 2014). Interpreting this null result cautiously, it might be possible that the current bottomup approach of manipulating physical stimulus aspects is less susceptible to influences of dispositional personality traits than previous experimental set-ups. To investigate this suggestion, future studies should assess the interplay of bottomup and top-down influences of social stimulus content and social context manipulations in within-subject designs. The processing advantage of social versus nonsocial feedback stimuli might be a rather universal effect that is not sensitive to self-construals, which have been shown to influence multiple cognitive and affective domains (Jiang, Varnum, Hou, \& Han, 2013; Lin, Lin, \& Han, 2008; Sui \& Han, 2007; Wang, Ma, \& Han, 2014).

Previous studies usually focused on top-down influence of social feedback by constructing ingenious experiments with cover stories in which participants were led to believe to directly receive feedback from others (Kujawa, Arfer, Klein, \& Proudfit, 2014; Somerville, Heatherton, \& Kelley, 2006). In contrast, the current study defines social feedback solely by its 
physical appearance, i.e., by the depiction of symbolic hand gestures. These gestures are used in face-to-face interactions on a regular basis to demonstrate favorable or unfavorable assessments by our conspecifics (Morris, 1994). Moreover, cross-cultural social media phenomena make use of exactly these gestures to convey social approval or disapproval. Both examples highlight that symbolic hand gestures as nonverbal signals are tightly linked to social interaction even in the absence of direct interaction partners. Thus, although the current study did not introduce an elaborate social setting, the sole use of pictures of social communication signals was strong enough to trigger rapid and spontaneous stimulus-driven saliency to take effect, thereby reflecting a processing advantage of social stimuli. Moreover, the stimuli used were matched for visual complexity, thereby minimizing the possibility that the observed effects were driven by other stimulus characteristics than the availability of social signals (Song et al., 2005).

Although the effects of sex/gender were not in the focus of the current study, we decided to address them in additional analyses and report the findings to provide a more exhaustive results overview_also including null results (see Eliot, 2011). In addition, van der Veen et al. (2016) suggested considering sex/gender as important individual difference when conducting research in the domain of social feedback. The authors observed differential sensitivity of their physiological markers in women and men when introducing a social evaluation situation. In contrast, the current study used a bottom-up approach to manipulate social feedback content, which was less susceptible to sex/gender differences. Only FM $\Theta$ oscillations differed in women and men when comparing overall effects of social versus nonsocial stimuli. Higher sensitivity to social versus nonsocial stimuli in women might reflect an evolutionary residue, socially acquired gender roles promoting social interaction, or a combination of both (Eagly, 2009). The interaction of biological sex and sociocultural gender roles during social cognition is far from understood and warrants future investigations (Pavlova, 2017). However, the observed FM $\Theta$ enhancement for social negative versus nonsocial negative feedback was not affected by sex/gender. This allows us to conclude that the current manipulation elicited rather comparable neural and behavioral results in all participants.

Our results imply that the use of hand gestures is an easy-to-use strategy to enhance the saliency of performance feedback stimuli. This could be relevant for clinical intervention and for neuropsychological rehabilitation programs. Middle-European and Chinese individuals showed comparable neural activation pattern when interpreting the hand gestures to adapt subsequent behavior. Future studies should assess large and culturally diverse samples to test whether the observed processing advantage of social stimuli is indeed independent of individuals' cultural backgrounds.

\section{Conclusions}

Using a bottom-up approach as experimental manipulation, we replicated the processing advantage of social over nonsocial complex stimuli during feedback processing in a Chinese sample. Not only did ERP components show amplitude enhancement for social compared with nonsocial stimuli, also neural oscillations in the theta range mirrored the previously observed activation pattern - in particular the differentiation between negative and positive feedback. This early processing advantage of social over nonsocial stimuli seems to be independent of cultural background or dispositional personality traits associated with enhanced social interaction. This further corroborates the notion that the dimension of social versus nonsocial characteristics should be considered as separate stimulus category that is culturally universal.

Acknowledgments This research was supported by the China Postdoctoral Science Foundation (2017M610670 to DMP) and the National Natural Science Foundation of China (projects 31661143039, 31470986, 31421003 to SH). The funding sources had no role in study design, data collection, analysis, or interpretation of the current data.

\section{Compliance with ethical standards}

Conflict of interest The authors report no conflicts of interest. The data are available online: https://osf.io/bq2cp/?view_only= 54a16f7852dc456cba20831b0f051cf0.

\section{References}

Alexander, W. H., \& Brown, J. W. (2011). Medial prefrontal cortex as an action-outcome predictor. Nature Neuroscience, 14(10), 1338-1344.

Baumeister, R. F., Vohs, K. D., DeWall, C. N., \& Zhang, L. (2007). How emotion shapes behavior: feedback, anticipation, and reflection, rather than direct causation. Pers Soc Psychol Rev, 11(2), 167-203. doi: https://doi.org/10.1177/1088868307301033

Bayer, M., Ruthmann, K., \& Schacht, A. (2017). The impact of personal relevance on emotion processing: evidence from event-related potentials and pupillary responses. Soc Cogn Affect Neurosci, 12(9), 1470-1479. doi: https://doi.org/10.1093/scan/nsx075

Bell, A. J., \& Sejnowski, T. J. (1995). An information-maximization approach to blind separation and blind deconvolution. Neural computation, 7(6), 1129-1159.

Bellebaum, C., \& Daum, I. (2008). Learning-related changes in reward expectancy are reflected in the feedback-related negativity. European Journal of Neuroscience, 27(7), 1823-1835.

Britton, J. C., Phan, K. L., Taylor, S. F., Welsh, R. C., Berridge, K. C., \& Liberzon, I. (2006). Neural correlates of social and nonsocial emotions: An fMRI study. Neuroimage, 31(1), 397-409. doi: https://doi. org/10.1016/j.neuroimage.2005.11.027

Cavanagh, J. F., \& Frank, M. J. (2014). Frontal theta as a mechanism for cognitive control. Trends Cogn Sci, 18(8), 414-421. doi: https://doi. org/10.1016/j.tics.2014.04.012

Cavanagh, J. F., \& Shackman, A. J. (2015). Frontal midline theta reflects anxiety and cognitive control: meta-analytic evidence. $J$ Physiol Paris, 109(1-3), 3-15. doi: https://doi.org/10.1016/j.jphysparis. 2014.04.003 
Cavanagh, J. F., Zambrano-Vazquez, L., \& Allen, J. J. (2012). Theta lingua franca: a common mid-frontal substrate for action monitoring processes. Psychophysiology, 49(2), 220-238. doi: https://doi.org/ 10.1111/j.1469-8986.2011.01293.x

Cohen, J. (1988). Statistical Power Analysis for the Behavioral Sciences.

Cohen, M. (2011). It's about Time. [Editorial]. Frontiers in Human Neuroscience, 5(2). doi: https://doi.org/10.3389/fnhum.2011.00002

Cohen, M. X., \& Donner, T. H. (2013). Midfrontal conflict-related thetaband power reflects neural oscillations that predict behavior. $J$ Neurophysiol, 110(12), 2752-2763. doi: https://doi.org/10.1152/jn. 00479.2013

Cohen, M. X., Elger, C. E., \& Ranganath, C. (2007). Reward expectation modulates feedback-related negativity and EEG spectra. Neuroimage, 35(2), 968-978. doi:https://doi.org/10.1016/j. neuroimage.2006.11.056

Cristofori, I., Moretti, L., Harquel, S., Posada, A., Deiana, G., Isnard, J., . . . Sirigu, A. (2013). Theta signal as the neural signature of social exclusion. Cerebral Cortex, 23(10), 2437-2447. doi: https://doi.org/10. 1093/cercor/bhs 236

Cuthbert, B. N., Schupp, H. T., Bradley, M. M., Birbaumer, N., \& Lang, P. J. (2000). Brain potentials in affective picture processing: Covariation with autonomic arousal and affective report. Biological Psychology, 52(2), 95-111.

Delorme, A., \& Makeig, S. (2004). EEGLAB: An open source toolbox for analysis of single-trial EEG dynamics including independent component analysis. Journal of Neuroscience Methods, 134(1), 9-21.

Dunbar, R. I. M. (1998). The social brain hypothesis. Evolutionary Anthropology: Issues, News, and Reviews, 6(5), 178-190. doi: https://doi.org/10.1002/(SICI)1520-6505(1998)6:5<178::AIDEVAN5>3.0.CO;2-8

Duncan Johnson, C. C., \& Donchin, E. (1977). On quantifying surprise. The variation of event related potentials with subjective probability. Psychophysiology, 14(5), 456-467.

Eagly, A. H. (2009). The his and hers of prosocial behavior: an examination of the social psychology of gender. Am Psychol, 64(8), 644-658. doi: https://doi.org/10.1037/0003-066x.64.8.644

Eliot, L. (2011). The trouble with sex differences. Neuron, 72(6), 895898. doi: https://doi.org/10.1016/j.neuron.2011.12.001

Enriquez-Geppert, S., Huster, R. J., Figge, C., \& Herrmann, C. S. (2014). Self-regulation of frontal-midline theta facilitates memory updating and mental set shifting. Front Behav Neurosci, 8, 420. doi: https:// doi.org/10.3389/fnbeh.2014.00420

Faul, F., Erdfelder, E., Buchner, A., \& Lang, A. G. (2009). Statistical power analyses using $\mathrm{G}^{*}$ Power 3.1: tests for correlation and regression analyses. Behavior Research Methods, 41(4), 1149-1160. doi: https://doi.org/10.3758/brm.41.4.1149

Gehring, W. J., \& Willoughby, A. R. (2002). The medial frontal cortex and the rapid processing of monetary gains and losses. Science, 295(5563), 2279-2282. doi: https://doi.org/10.1126/science. 1066893

Giner-Sorolla, R. (2012). Science or Art? How Aesthetic Standards Grease the Way Through the Publication Bottleneck but Undermine Science. Perspectives on Psychological Science, 7(6), 562-571. doi: https://doi.org/10.1177/1745691612457576

Han, S. (2017). The sociocultural brain - a cultural neuroscience approach to human nature. Oxford: Oxford University Press.

Han, S., Northoff, G., Vogeley, K., Wexler, B. E., Kitayama, S., \& Varnum, M. E. (2013). A cultural neuroscience approach to the biosocial nature of the human brain. Annu Rev Psychol, 64, 335-359. doi: https://doi.org/10.1146/annurevpsych-071112-054629

Hariri, A. R., Tessitore, A., Mattay, V. S., Fera, F., \& Weinberger, D. R. (2002). The amygdala response to emotional stimuli: a comparison of faces and scenes. Neuroimage, 17(1), 317-323.
Hayden, B. Y., Heilbronner, S. R., Pearson, J. M., \& Platt, M. L. (2011). Surprise signals in anterior cingulate cortex: neuronal encoding of unsigned reward prediction errors driving adjustment in behavior. Journal of Neuroscience, 31(11), 4178-4187. doi: https://doi.org/10. 1523/jneurosci.4652-10.2011

Hitokoto, H., Glazer, J., \& Kitayama, S. (2016). Cultural shaping of neural responses: Feedback-related potentials vary with selfconstrual and face priming. Psychophysiology, 53(1), 52-63. doi: https://doi.org/10.1111/psyp.12554

Holroyd, C. B., Nieuwenhuis, S., Yeung, N., \& Cohen, J. D. (2003). Errors in reward prediction are reflected in the event-related brain potential. NeuroReport, 14(18), 2481-2484.

Jiang, C., Varnum, M. E., Hou, Y., \& Han, S. (2013). Distinct effects of self-construal priming on empathic neural responses in Chinese and Westerners. Social Neuroscience. doi: https://doi.org/10.1080/ 17470919.2013.867899

Keselman, H. J., Wilcox, R. R., \& Lix, L. M. (2003). A generally robust approach to hypothesis testing in independent and correlated groups designs. Psychophysiology, 40(4), 586-596.

Kirk, R. E. (1996). Practical Significance: A Concept Whose Time Has Come. Educational and Psychological Measurement, 56(5), 746759. doi: https://doi.org/10.1177/0013164496056005002

Kitayama, S., \& Park, J. (2014). Error-related brain activity reveals selfcentric motivation: Culture matters. Journal of Experimental Psychology: General, 143(1), 62-70. doi: https://doi.org/10.1037/ a0031696

Kogler, L., Sailer, U., Derntl, B., \& Pfabigan, D. M. (2017). Processing expected and unexpected uncertainty is modulated by fearlessdominance personality traits - An exploratory ERP study on feedback processing. Physiol Behav, 168, 74-83. doi: https://doi.org/10. 1016/j.physbeh.2016.10.016

Kujawa, A., Arfer, K. B., Klein, D. N., \& Proudfit, G. H. (2014). Electrocortical reactivity to social feedback in youth: a pilot study of the Island Getaway task. Dev Cogn Neurosci, 10, 140-147. doi: https://doi.org/10.1016/j.den.2014.08.008

Kutas, M., McCarthy, G., \& Donchin, E. (1977). Augmenting mental chronometry: the P300 as a measure of stimulus evaluation time. Science, 197(4305), 792-795.

$\mathrm{Li}, \mathrm{X}$. (1983). The distribution of left and right handedness in chinese people. Acta Psychologica Sinica, 15(03), 27-35.

Lin, Z., Lin, Y., \& Han, S. (2008). Self-construal priming modulates visual activity underlying global/local perception. Biological Psychology, 77(1), 93-97. doi:Export Date 22 January 2014

Liu, Y., Nelson, L. D., Bernat, E. M., \& Gehring, W. J. (2014). Perceptual properties of feedback stimuli influence the feedback-related negativity in the flanker gambling task. Psychophysiology, 51(8), 782788. doi: https://doi.org/10.1111/psyp. 12216

Luck, S. J., \& Gaspelin, N. (2017). How to get statistically significant effects in any ERP experiment (and why you shouldn't). Psychophysiology, 54(1), 146-157. doi: https://doi.org/10.1111/ psyp.12639

Ma, Y., Bang, D., Wang, C., Allen, M., Frith, C., Roepstorff, A., \& Han, S. (2014). Sociocultural patterning of neural activity during selfreflection. Soc Cogn Affect Neurosci, 9(1), 73-80. doi: https://doi. org/10.1093/scan/nss 103

Makeig, S., Debener, S., Onton, J., \& Delorme, A. (2004). Mining eventrelated brain dynamics. Trends in Cognitive Sciences, 8(5), 204-210. doi: doi:https://doi.org/10.1016/j.tics.2004.03.008

Markus, H. R., \& Kitayama, S. (1991). Culture and the self: Implications for cognition, emotion, and motivation. [doi:10.1037/0033295X.98.2.224]. Psychological Review, 98, 224-253. doi: https:// doi.org/10.1037/0033-295x.98.2.224

Miltner, W. H. R., Braun, C. H., \& Coles, M. G. H. (1997). Event-related brain potentials following incorrect feedback in a time-estimation task: Evidence for a 'generic' neural system for error detection. Journal of Cognitive Neuroscience, 9(6), 788-798. 
Morris, D. (1994). Bodytalk: The Meaning of Human Gestures. Crown Publishing Group.

Nieuwenhuis, S., Aston-Jones, G., \& Cohen, J. D. (2005). Decision making, the P3, and the locus coeruleus-norepinephrine system. Psychol Bull, 131(4), 510-532. doi: https://doi.org/10.1037/0033-2909.131. 4.510

Norris, C. J., Chen, E. E., Zhu, D. C., Small, S. L., \& Cacioppo, J. T. (2004). The interaction of social and emotional processes in the brain. J Cogn Neurosci, 16(10), 1818-1829. doi: https://doi.org/10. $1162 / 0898929042947847$

Okruszek, L., Wichniak, A., Jarkiewicz, M., Schudy, A., Gola, M., Jednorog, K., . . . Lojek, E. (2016). Social and nonsocial affective processing in schizophrenia - An ERP study. Int J Psychophysiol, 107, 54-62. doi: https://doi.org/10.1016/j.jpsycho.2016.06.007

Open Science, C. (2015). PSYCHOLOGY. Estimating the reproducibility of psychological science. Science, 349(6251), aac4716. doi: https:// doi.org/10.1126/science.aac4716

Osinsky, R., Seeger, J., Mussel, P., \& Hewig, J. (2016). Face-induced expectancies influence neural mechanisms of performance monitoring. Cogn Affect Behav Neurosci, 16(2), 261-275. doi: https://doi. org/10.3758/s13415-015-0387-y

Pashler, H., \& Harris, C. R. (2012). Is the Replicability Crisis Overblown? Three Arguments Examined. Perspectives on Psychological Science, 7(6), 531-536. doi: https://doi.org/10.1177/ 1745691612463401

Pavlova, M. A. (2017). Sex and gender affect the social brain: Beyond simplicity. J Neurosci Res, 95(1-2), 235-250. doi: https://doi.org/10. 1002/jnr.23871

Pfabigan, D. M., Alexopoulos, J., Bauer, H., Lamm, C., \& Sailer, U. (2011a). All about the money? External performance monitoring is affected by monetary, but not by socially conveyed feedback cues in more antisocial individuals. [Original Research]. Frontiers in Human Neuroscience, 5. doi: https://doi.org/10.3389/fnhum.2011. 00100

Pfabigan, D. M., Alexopoulos, J., Bauer, H., \& Sailer, U. (2011b). Manipulation of feedback expectancy and valence induces negative and positive reward prediction error signals manifest in event-related brain potentials. Psychophysiology, 48(5), 656-664.

Pfabigan, D. M., Gittenberger, M., \& Lamm, C. (2019). Social dimension and complexity differentially influence brain responses during feedback processing. Social neuroscience, 14:1, 26-40. doi: https://doi. org/10.1080/17470919.2017.1395765

Pfabigan, D. M., Sailer, U., \& Lamm, C. (2015). Size does matter! Perceptual stimulus properties affect event-related potentials during feedback processing. Psychophysiology, 52(9), 1238-1247. doi: https://doi.org/10.1111/psyp.12458

Pfabigan, D. M., Wucherer, A. M., Wang, X., Pan, X., Lamm, C., \& Han, S. (2018). Cultural influences on the processing of social comparison feedback signals-an ERP study. Soc Cogn Affect Neurosci, 13(12), 1317-1326. doi: https://doi.org/10.1093/scan/nsy097

Pfabigan, D. M., Zeiler, M., Lamm, C., \& Sailer, U. (2014). Blocked versus randomized presentation modes differentially modulate feedback-related negativity and P3b amplitudes. Clinical Neurophysiology, 125(4), 715-726. doi: https://doi.org/10.1016/j. clinph.2013.09.029

Pfurtscheller, G., \& Lopes da Silva, F. H. (1999). Event-related EEG/ MEG synchronization and desynchronization: basic principles. Clin Neurophysiol, 110(11), 1842-1857.

Polich, J. (2007). Updating P300: An integrative theory of P3a and P3b. Clinical Neurophysiology, 118(10), 2128-2148.

Porges, S. W. (2003) Social Engagement and Attachment: A Phylogenetic Perspective. Vol. 1008. Ann N Y Acad Sci (pp. 31-47).

Potts, G. F. (2004). An ERP index of task relevance evaluation of visual stimuli. Brain Cogn, 56(1), 5-13. doi: https://doi.org/10.1016/j. bandc.2004.03.006
Potts, G. F., Martin, L. E., Burton, P., \& Montague, P. R. (2006). When things are better or worse than expected: The medial frontal cortex and the allocation of processing resources. Journal of Cognitive Neuroscience, 18(7), 11121119.

Rauchbauer, B., Majdandžić, J., Hummer, A., Windischberger, C., \& Lamm, C. (2015). Distinct neural processes are engaged in the modulation of mimicry by social group-membership and emotional expressions. Cortex, 70, 49-67. doi:https://doi.org/10.1016/j.cortex. 2015.03.007

Rousselet, G. A., \& Pernet, C. R. (2012). Improving standards in brain-behaviour correlation analyses. [Perspective]. Frontiers in Human Neuroscience, 6. doi: https://doi.org/10.3389/ fnhum.2012.00119

Schacht, A., \& Vrticka, P. (2018). Spatiotemporal pattern of appraising social and emotional relevance: Evidence from event-related brain potentials. Cogn Affect Behav Neurosci. doi: https://doi.org/10. 3758/s13415-018-0629-x

Scharpf, K. R., Wendt, J., Lotze, M., \& Hamm, A. O. (2010). The brain's relevance detection network operates independently of stimulus modality. Behav Brain Res, 210(1), 16-23. doi: https://doi.org/10.1016/ j.bbr.2010.01.038

Scherer, K. R. (2009). Emotions are emergent processes: they require a dynamic computational architecture. Philos Trans $R$ Soc Lond $B$ Biol Sci, 364(1535), 3459-3474. doi: https://doi.org/10.1098/rstb. 2009.0141

Schmidt, S. (2009). Shall we really do it again? the powerful concept of replication is neglected in the social sciences. Review of General Psychology, 13(2), 90-100. doi: https:// doi.org/10.1037/a0015108

Singelis, T. M. (1994). The measurement of independent and interdependent self-construals. Personality and Social Psychology Bulletin, 20(5), 580-591. doi: https://doi.org/10. 1177/0146167294205014

Somerville, L. H., Heatherton, T. F., \& Kelley, W. M. (2006). Anterior cingulate cortex responds differentially to expectancy violation and social rejection. Nat Neurosci, 9(8), 1007-1008. doi: https://doi.org/ $10.1038 / \mathrm{nn} 1728$

Song, Y., Ding, Y., Fan, S., Qu, Z., Xu, L., Lu, C., \& Peng, D. (2005). Neural substrates of visual perceptual learning of simple and complex stimuli. Clin Neurophysiol, 116(3), 632-639. doi: https://doi. org/10.1016/j.clinph.2004.09.019

Sui, J., \& Han, S. (2007). Self-construal priming modulates neural substrates of self-awareness. Psychological Science, 18(10), 861-866. doi: Export Date 22 January 2014

Talmi, D., Atkinson, R., \& El-Deredy, W. (2013). The feedback-related negativity signals salience prediction errors, not reward prediction errors. J Neurosci, 33(19), 8264-8269. doi: https://doi.org/10.1523/ jneurosci.5695-12.2013

van der Molen, M. J., Dekkers, L. M., Westenberg, P. M., van der Veen, F. M., \& van der Molen, M. W. (2016). Why don't you like me? Midfrontal theta power in response to unexpected peer rejection feedback. Neuroimage. doi: https://doi.org/10.1016/j.neuroimage. 2016.08.045

van der Veen, F. M., van der Molen, M. J., van der Molen, M. W., \& Franken, I. H. (2016). Thumbs up or thumbs down? Effects of neuroticism and depressive symptoms on psychophysiological responses to social evaluation in healthy students. Cogn Affect Behav Neurosci, 16(5), 836-847. doi: https://doi.org/10.3758/ s13415-016-0435-2

van Noordt, S. J., White, L. O., Wu, J., Mayes, L. C., \& Crowley, M. J. (2015). Social exclusion modulates eventrelated frontal theta and tracks ostracism distress in children. Neuroimage, 118, 248-255. doi: https://doi.org/10.1016/j. neuroimage.2015.05.085 
Vrtička, P., Sander, D., \& Vuilleumier, P. (2011). Effects of emotion regulation strategy on brain responses to the valence and social content of visual scenes. Neuropsychologia, 49(5), 1067-1082. doi: https://doi.org/10.1016/j.neuropsychologia.2011.02.020

Wagner, D. D., Kelley, W. M., Haxby, J. V., \& Heatherton, T. F. (2016). The Dorsal Medial Prefrontal Cortex Responds Preferentially to Social Interactions during Natural Viewing. J Neurosci, 36(26), 6917-6925. doi: https://doi.org/10.1523/jneurosci.4220-15.2016

Wang, C., Ma, Y., \& Han, S. (2014). Self-construal priming modulates pain perception: Event-related potential evidence. Cogn Neurosci, 5(1), 3-9. doi: https://doi.org/10.1080/17588928.2013.797388
Wilcox, R. R. (2010). Fundamentals of modern statistical methods: Substantially improving power and accuracy. New York: Springer.

Wilcox, R. R. (2012). Modern statistics for the social and behavioral sciences: A practical introduction. Boca Raton: CRC Press.

Publisher's note Springer Nature remains neutral with regard to jurisdictional claims in published maps and institutional affiliations. 